Hyl I er aas and Ki noshi ta wave f unct i ons: Revi si on and cor recti on

\begin{tabular}{|c|c|}
\hline 著者 & KOGA Toshi kat su \\
\hline $\begin{array}{l}\text { jour nal or } \\
\text { publ i cat } i \text { on } \mathrm{titl} e\end{array}$ & The j our nal of chemical physi cs \\
\hline vol une & 94 \\
\hline nunber & 8 \\
\hline page $r$ ange & $5530-5532$ \\
\hline year & 1991-04 15 \\
\hline URL & ht t p: //hdl . handl e. net /10258/857 \\
\hline
\end{tabular}




\title{
Hylleraas and Kinoshita wave functions: Revision and correction
}

\author{
Toshikatsu Koga \\ Department of Applied Chemistry, Muroran Institute of Technology, Muroran, Hokkaido 050, Japan
}

(Received 4 December 1990; accepted 31 December 1990)

\begin{abstract}
As is the case of the Hylleraas six-term wave function, it is shown that several Hylleraas and Kinoshita wave functions for two-electron atoms given in the literature are not sufficiently accurate particularly in their exponents $\xi$. For example, Kinoshita reported that his 80-term helium wave function has the minimum energy of -2.9037237 a.u. for $\zeta=1.855199$, while we obtain -2.903724347 a.u. for $\zeta=2.245896$.
\end{abstract}

In a very recent paper, ${ }^{1}$ it has been reported that the known parameter values ${ }^{2-5}$ for the familiar Hylleraas sixterm wave function ${ }^{3-9}$ for the helium atom,

$\Psi_{6}=\exp (-\zeta s)\left(1+c_{1} u+c_{2} t^{2}+c_{3} s+c_{4} s^{2}+c_{5} u^{2}\right)$,

are not as accurate as previously believed, and accordingly the wave function (1) can have an energy lower than the value hitherto given in the literature ${ }^{3-9}$ by about 0.0001 . (Atomic units are used throughout.) Comparison of the new and literature parameters has shown' that the essential origin of the energy improvement lies in the value of the exponent $\zeta$, and sufficient optimization of this nonlinear parameter has been suggested to be crucial for the accurate determination of the wave function and the associated energy.

Stimulated by the above result, we have considered it necessary to reconfirm other (more elaborate) Hylleraas wave functions reported in the literature, ${ }^{10-12}$ since they are often used as parent functions to derive physical quantities of near exact accuracy (see, e.g., Refs. 13 and 14). Similar examination has also been carried out for the Kinoshita wave functions. ${ }^{15,16}$ Unfortunately, we have to report in this article that the known parameters and associated energies are insufficiently accurate for both the Hylleraas and Kinoshita wave functions.

The Hylleraas and Kinoshita wave functions for ground state two-electron atoms are generally given by

$$
\begin{aligned}
& \Psi_{N}=\exp (-\zeta s) \sum_{i=1}^{N} c_{i} s^{l^{1} t} t^{2 m_{i}} u^{n_{i}}, \\
& \Psi_{N}=\exp (-\zeta s) \sum_{i=1}^{N} c_{i} s^{l_{i}}(u / s)^{m_{i}}(\dot{t} / u)^{2 n_{i}},
\end{aligned}
$$

respectively, where $s=r_{1}+r_{2}, t=r_{1}-r_{2}$, and $u=r_{12}$ are Hylleraas coordinates, $\left\{l_{i}, m_{i}, n_{i}\right\}$ are non-negative integers, and $\zeta$ and $\left\{c_{i}\right\}$ are variational parameters.

The two-electron atomic Hamiltonian in the Hylleraas coordinates is given elsewhere. ${ }^{2,6,7,17}$ For the trial functions (2a) and (2b), we can express all the Hamiltonian $H_{i j}$ and overlap $S_{i j}$ matrix elements in terms of a basic integral (cf. Ref. 11),

$$
\begin{aligned}
\int_{0}^{\infty} d s & \int_{0}^{s} d t \int_{t}^{s} d u \exp (-2 \zeta s) s^{I} t^{J} u^{K} \\
= & (I+J+K+2) ! /\left[(2 \zeta)^{I+J+K+3}\right. \\
& \times(J+1)(J+K+2)] .
\end{aligned}
$$

The variation of the energy expression $E=\mathbb{C}^{+} \mathbb{H C} / \mathbb{C}^{+} S \mathbb{C}$ with respect to the linear parameters $\left\{c_{i}\right\}$ results in an eigenvalue equation $\mathbb{H C}=\mathrm{SCE}$. Then using an appropriate routine program of the generalized eigenvalue equation solver, ${ }^{18}$ we can accurately determine the energy $E$ and the expansion coefficients $\left\{c_{i}\right\}$ for a given value of $\xi$. For the determination

\begin{tabular}{|c|c|c|c|c|c|}
\hline \multirow[b]{2}{*}{$N$} & \multicolumn{3}{|c|}{ Literature } & \multicolumn{2}{|c|}{ Present } \\
\hline & $E$ & $\zeta$ & Ref. & $E$ & $\zeta$ \\
\hline 6 & -2.90324 & 1.82 & 2 & $-2.903329354^{\mathrm{a}}$ & $1.755656^{\mathrm{a}}$ \\
\hline 10 & -2.9036027 & 1.755013 & 10 & -2.903602729 & 1.757763 \\
\hline 14 & -2.903701 & 1.90 & 11 & -2.903701491 & 1.897917 \\
\hline 18 & $\begin{array}{l}-2.90371^{\mathrm{b}} \\
-2.9037150\end{array}$ & $\begin{array}{l}1.925 \\
1.944606\end{array}$ & $\begin{array}{l}11 \\
15\end{array}$ & -2.903716636 & 1.938541 \\
\hline 20 & $\begin{array}{l}-2.9037179 \\
\left(-2.903717733^{\mathrm{c}}\right)\end{array}$ & 1.935 & 12 & -2.903717754 & 1.932909 \\
\hline
\end{tabular}
of the nonlinear parameter $\zeta$, we have employed two different methods in order to check its optimality and accuracy. One method finds the optimum $\zeta$ by the minimization of $E$

TABLE I. Energy $E$ and exponent $\zeta$ of several Hylleraas wave functions of the helium atom.

\footnotetext{
${ }^{a}$ Reference 1 .

${ }^{\mathrm{b}}$ In Ref. 11, the energy -2.903716 is also given for the 18-term function. However, it is not included in this table since no corresponding wave function is reported.
}

"Recalculated based on the parameters given in Ref. 12. 
TABLE II. The Hyllerass 20-term energy for the helium atom as a function of the exponent $\zeta$ around its optimum value. The linear parameters $\left\{c_{i}\right\}$ are optimized for each $\zeta$ value.

\begin{tabular}{ccc}
\hline \hline$\zeta$ & $E$ & $-V / T$ \\
\hline 1.925 & -2.903717736 & 2.000003108 \\
1.926 & -2.903717740 & 2.000002729 \\
1.927 & -2.903717744 & 2.000002346 \\
1.928 & -2.903717747 & 2.000001959 \\
1.929 & -2.903717750 & 2.000001568 \\
1.930 & -2.903717752 & 2.000001173 \\
1.931 & -2.903717753 & 2.000000774 \\
1.932 & -2.903717754 & 2.000000370 \\
$1.933^{\mathrm{a}}$ & -2.903717754 & 1.999999963 \\
1.934 & -2.903717754 & 1.999999551 \\
$1.935^{\mathrm{b}}$ & -2.903717753 & 1.999999135 \\
1.936 & -2.903717751 & 1.999998714 \\
1.937 & -2.903717749 & 1.999998290 \\
1.938 & -2.903717746 & 1.999997861 \\
1.939 & -2.903717743 & 1.999997427 \\
1.940 & -2.903717739 & 1.999996990 \\
\hline \hline
\end{tabular}

a This $\zeta$ is closest to the present optimum value in this table.

${ }^{b}$ The $\zeta$ value of Hart and Herzberg (Ref. 12).

based on the Powell method of conjugate directions. ${ }^{19}$ The other method determines the parameter $\xi$ iteratively so that the virial theorem ${ }^{20}$ is satisfied, i.e., $V=-2 T$ or $E=-V^{2} / 4 T$ holds, where $T$ and $V$ are the kinetic and potential energy components. (Note that in the above procedures of $\xi$ optimization, all the $\left\{c_{i}\right\}$ are variationally determined for each value of $\xi$.)

Table I compares the present results for the Hylleraas 10-, 14-, 18-, and 20-term wave functions with those reported by Herzberg and co-workers. ${ }^{10-12}$ (The $\left\{c_{i}\right\}$ are omitted there, but they are available upon request.) The energies for $N=10$ and 14 in the literature agree with the present values within the given decimal places. A non-negligible improvement is found for the energy of $N=18$. In the case of $N=20$, however, the literature energy value ${ }^{12}$ is lower than that we obtained. We were not able to reproduce the literature value even when we input the corresponding parameters reported in Ref. 12: It is suggested that the energy interpolation used in the literature is not sufficiently accurate. In Table II, we have explicitly given the minimum energy as a function of the exponent $\xi$ around its optimun value. The very weak dependence of the energy upon this parameter is observed.

TABLE III. Energy $E$ and exponent $\zeta$ of several Kinoshita wave functions for the helium atom.

\begin{tabular}{|c|c|c|c|c|c|}
\hline \multirow[b]{2}{*}{$N$} & \multicolumn{3}{|c|}{ Literature } & \multicolumn{2}{|l|}{ Present } \\
\hline & $E$ & $\xi$ & Ref. & $E$ & $\zeta$ \\
\hline 10 & -2.9036261 & 1.729575 & 15 & -2.903627336 & 1.745726 \\
\hline 22 & -2.9037142 & 1.853910 & 15 & -2.903718784 & 1.812833 \\
\hline 34 & -2.9037223 & 1.856694 & 15 & -2.903722780 & 2.021305 \\
\hline 38 & -2.9037225 & 1.860237 & 15 & -2.903722839 & 2.028535 \\
\hline 39 & -2.9037225 & 1.860556 & 15 & -2.903722910 & 1.996338 \\
\hline 80 & -2.9037237 & 1.855199 & 16 & -2.903724347 & 2.245896 \\
\hline
\end{tabular}

TABLE IV. The Kinoshita 80-term energy for the helium atom as a function of the exponent $\zeta$ around its optimum value. The linear parameters $\left\{c_{i}\right\}$ are optimized for each $\zeta$ value.

\begin{tabular}{ccc}
\hline \hline$\zeta$ & $E$ & $-V / T$ \\
\hline 1.80 & -2.903724265 & 2.000000263 \\
1.82 & -2.903724273 & 2.000000244 \\
1.84 & -2.903724280 & 2.000000226 \\
$1.86^{\mathrm{a}}$ & -2.903724287 & 2.000000210 \\
1.88 & -2.903724293 & 2.000000195 \\
1.90 & -2.903724299 & 2.000000182 \\
1.92 & -2.903724305 & 2.000000169 \\
1.94 & -2.903724309 & 2.000000157 \\
1.96 & -2.903724314 & 2.000000146 \\
1.98 & -2.903724318 & 2.000000136 \\
2.00 & -2.903724322 & 2.000000127 \\
2.02 & -2.903724325 & 2.000000118 \\
2.04 & -2.903724329 & 2.000000109 \\
2.06 & -2.903724332 & 2.000000101 \\
2.08 & -2.903724334 & 2.000000094 \\
2.10 & -2.903724337 & 2.000000086 \\
2.12 & -2.903724339 & 2.000000079 \\
2.14 & -2.903724341 & 2.000000070 \\
2.16 & -2.903724343 & 2.000000062 \\
2.18 & -2.903724345 & 2.000000051 \\
2.20 & -2.903724346 & 2.000000039 \\
2.22 & -2.903724347 & 2.000000025 \\
$2.24^{b}$ & -2.903724347 & 2.000000006 \\
2.26 & -2.903724347 & 1.999999983 \\
2.28 & -2.903724346 & 1.999999954 \\
2.30 & -2.903724344 & 1.999999916 \\
\hline \hline
\end{tabular}

"This $\zeta$ value is closest to the Kinoshita value (Ref. 16) in this table.

${ }^{\mathrm{b}}$ This $\zeta$ value is closest to the present optimum value in this table.

Though the change in the energy value is relatively small, the optimum values for the nonlinear parameter $\zeta$ are found to be considerably different except for the 14-term function. The difference in the exponent $\zeta$ implies the difference in the coefficients $\left\{c_{i}\right\}$, and hence a nontrivial change in the wave function $\Psi_{N}$. Since the change in the wave function linearly affects the resultant physical properties other than the total energy $E$, we expect a significant change in the calculated properties. For example, Benesch ${ }^{14}$ constructed the radial electron density $D(r)$ based on the 20-term function reported by Hart and Herzberg ${ }^{12}$ in the form of

$$
D(r)=\exp (-2 \zeta r) \sum_{i} a_{i} r^{i}+\exp (-4 \zeta r) \sum_{i} b_{i} r^{i} .
$$

For the $\mathrm{He}$ atom, his first three $a_{i}$ 's are 3.316169 , 40.657413 , and -8.027578 , but the present wave function gives $3.304513,40.668878$, and -8.196001 . He also reported $^{14}$ the electron-nuclear cusp constant $C_{\mathrm{EN}}$ $=2.002967$ and the virial ratio ${ }^{21}-V / T=1.999998869$, but we have $C_{\mathrm{EN}}=2.002765$ and $-V / T=2.000000000$ from the present 20 -term wave function. (The exact values are $C_{\mathrm{EN}}=-V / T=2$ for the He atom.)

Our results for the several Kinoshita wave functions are summarized in Table III in comparison with the literature values. ${ }^{15,16}$ The energies are seen to be improved for all cases, particularly for the 10- and 22-term wave functions. We also find that the Kinoshita 80-term function has an energy much closer to the "exact" value $22,23-2.903724377$ than hitherto believed. In contrast to the rather small change in the energy, the difference in the optimum exponent $\zeta$ is quite 
remarkable. Table IV shows the explicit dependence of the energy upon the exponent $\zeta$ for the Kinoshita 80-term wave function. As has been exemplified for the Hylleraas 20-term wave function, we anticipate that the change in the wave function will give a nontrivial influence to the calculated physical properties.

We hope that the present report will be of some help to the future studies on two-electron atoms based on the Hylleraas- and Kinoshita-type wave functions.

Note added in proof. After this paper was accepted for publication, Professor A. J. Thakkar kindly informed me that P. Jolly [Int. J. Quantum Chem. 16, 1149 (1979)] had reported a similar improvement of the Hylleraas wave function for the helium six-term case.

'T. Koga, J. Chem. Phys. 93, 3720 (1990).

'E. A. Hylleraas, Z. Phys. 54, 347 (1929).

${ }^{3}$ L. Pauling and E. B. Wilson, Jr., Introduction to Quantum Mechanics (McGraw-Hill, New York, 1935), p. 224.

${ }^{4} \mathrm{~J}$. C. Slater, Quantum Theory of Atomic Structure (McGraw-Hill, New York, 1960), Vol. 2, p. 36.
${ }^{5}$ J. C. Slater, Quantum Theory of Matter (McGraw-Hill, New York, 1968), p. 436.

${ }^{6}$ E. A. Hylleraas, Adv. Quantum Chem. 1, 1 (1964).

${ }^{7}$ E. A. Hylleraas, Mathematical and Theoretical Physics (Wiley-Interscience, New York, 1970), Vol. 2, p. 416ff.

${ }^{8} \mathrm{H}$. F. Hameka, Introduction to Quantum Theory (Harper and Row, New York, 1967).

${ }^{9} \mathrm{~F}$. L. Pilar, Elementary Quantum Chemistry (McGraw-Hill, New York, 1968), p. 244.

${ }^{10}$ S. Chandrasekhar, D. Elbery, and G. Herzberg, Phys. Rev. 91, 1172 (1953).

"'S. Chandrasekhar and G. Herzberg, Phys. Rev. 98, 1050 (1955).

12J. F. Hart and G. Herzberg, Phys. Rev. 106, 79 (1957).

${ }^{13}$ R. F. Curl, Jr. and C. A. Coulson, Proc. Phys. Soc. 85, 647 (1965).

${ }^{14}$ R. Benesch, J. Phys. B 4, 1403 (1971).

${ }^{15}$ T. Kinoshita, Phys. Rev. 105, 1490 (1957).

${ }^{16}$ T. Kinoshita, Phys. Rev. 115, 366 (1959).

${ }^{17}$ H. A. Bethe and E. E. Salpeter, Quantum Mechanics of One- and TwoElectron Atoms (Plenum, New York, 1977), p. 149.

${ }^{18}$ In the present study, the generalized eignevalue problem is solved by the Choleski decomposition followed by Householder's method.

${ }^{19}$ M. J. D. Powell, Comput. J. 7, 155 (1964).

${ }^{20}$ See, for example, P. -O. Löwdin, J. Mol. Spectrosc. 3, 46 (1959).

${ }^{21}$ The original virial ratio given by Benesch (Ref. 14) is inaccurate, since he obtained $T$ by subtracting $V$ from an inaccurate $E$.

${ }^{22}$ K. Frankowski and C. L. Pekeris, Phys. Rev. 146, 46 (1966).

${ }^{23}$ J. D. Baker, D. E. Freund, R. N. Hill, and J. D. Morgan III, Phys. Rev. A 41, 1247 (1990). 